\title{
Uso de moderadores de apetite entre estudantes da área da saúde na Região Sul do Brasil
}

\author{
The use of appetite suppressants among health sciences undergraduate \\ students in Southern Brazil \\ Carlos Zubaran ${ }^{1}$, Rubia Lazzaretti ${ }^{2}$
}

\section{RESUMO}

Objetivo: Estabelecer a prevalência do uso de moderadores de apetite entre estudantes da área da saúde na Região Sul do Brasil. Métodos: Estudantes universitários $(n=300)$ de sete cursos da área da saúde da Universidade de Caxias do Sul completaram um questionário sobre 0 uso de moderadores de apetite. Resultados: Uma significativa porcentagem da amostra (15\%; $n=45$ ) usou moderadores de apetite pelo menos uma vez na vida. As substâncias mais comumente usadas foram drogas estimulantes (5\%), incluindo amfepramona $(3,3 \%)$ e fenproporex (1,7\%). 0 uso de moderadores de apetite foi mais prevalente entre estudantes de Enfermagem (26,7\%) e Nutrição (24,4\%\%). Não foi relatado o uso de moderadores de apetite entre estudantes de Medicina. 0 uso de anorexígenos foi significativamente mais prevalente entre mulheres. A maioria dos que usaram supressores de apetite o fez sob orientação médica, como também usou tais substâncias por mais de 3 meses. Conclusão: 0 uso durante a vida de moderadores de apetite foi significativo entre estudantes da área da saúde, especialmente entre os matriculados em Enfermagem e Nutrição. As drogas estimulantes simpatomiméticas foram os agentes mais comumente usados.

Descritores: Depressores do apetite; Obesidade; Estudantes; Brasil

\section{ABSTRACT}

Objective: To investigate the prevalence of appetite suppressant use among health sciences students in Southern Brazil. Methods: Undergraduate students $(n=300)$ from seven health science undergraduate courses of the Universidade de Caxias do Sul completed a questionnaire about the use of substances to suppress appetite. Results: A significant percentage (15\%; $n=45)$ of research participants used appetite suppressants at least once in their lives. The most commonly used substances were sympathomimetic stimulant drugs (5\%), including amfepramone (3.3\%) and fenproporex (1.7\%). The lifetime use of appetite suppressants was more prevalent among Nursing $(26.7 \%)$ and Nutrition $(24.4 \% \%)$ students. There was no reported use of appetite suppressants among medical students. The use of appetite suppressants was significantly more prevalent among women. The majority of those who used these substances did so under medical recommendation. Most of users took appetite suppressants for more than 3 months. Conclusion: Lifetime use of appetite suppressants was substantial, being sympathomimetic stimulant drugs the most commonly used agents. Students enrolled in Nursing and Nutrition courses presented a significantly higher prevalence of lifetime use of appetite suppressants.

Keywords: Appetite suppressants; Obesity; Students; Brazil

\section{INTRODUÇÃO}

A obesidade é um dos distúrbio crônico mais comum nas sociedades industrializadas. $\mathrm{O}$ impacto da obesidade, em termos de saúde pública, tem sido largamente reconhecido $^{(1)}$. Atualmente, a comunidade médica define a obesidade como disfunção com patogênese multifatorial, que produz mudanças sistemáticas no estilo de vida e, na maioria dos casos graves, exige tratamento medicamentoso $^{(2)}$. Diversas classes de medicamentos estão disponíveis para combater a obesidade ${ }^{(3)}$. Recentemente, houve um aumento considerável do uso não médico e do abuso na prescrição de medicamentos para obesidade $^{(4)}$. O uso crônico de moderadores de apetite pode estar associado ao alto risco de distúrbios devido ao uso dessas substâncias ${ }^{(5)}$.

\footnotetext{
Trabalho realizado na Universidade de Caxias do Sul, Caxias do Sul, RS, Brasil.

${ }^{1}$ School of Medicine, University of Western Sydney, Sydney, Austrália; The Biological and Health Sciences Center, Universidade de Caxias do Sul, Caxias do Sul, RS, Brasil.

${ }^{2}$ The Biological and Health Sciences Center, Universidade de Caxias do Sul, Caxias do Sul, RS, Brasil.

Autor correspondente: Carlos Zubaran - Departamento de Psiquiatria-West Area Health Service - Caixa Postal 6010, Blacktown - NSW 2148 - Sydney (SYD), Austrália - Tel.: (+61 2) $9881-8888$ Fax: (+61 2) 9881- 8899 - E-mail: zubaran_jr@yahoo.com

Data de submissão: 20/6/2012 - Data de aceite: 4/2/2013

Conflito de interesse: não há.
} 
$\mathrm{O}$ abuso de moderadores de apetite tem sido frequentemente relatado como problemática em países em desenvolvimento ${ }^{(6-8)}$. Um levantamento conduzido em 1999 na cidade de São Paulo (SP), a maior cidade brasileira, revelou prevalência, durante a vida, de $0,9 \%$ do uso de moderadores de apetite ${ }^{(9)}$. Pesquisas conduzidas entre 1996 e 2001 com graduandos da Universidade de São Paulo (USP) revelaram aumento significante do uso de anfetaminas durante a vida (de 4,8 a 9,0\%), além do uso em 12 meses e nos últimos 30 dias $^{(10)}$.

$\mathrm{O}$ uso de moderadores de apetite no Brasil é mais prevalente entre as mulheres, para quem é geralmente correlacionado com cultura da magreza, considerada símbolo de beleza ${ }^{(6)}$. Evidências de estudos em diferentes regiões do Brasil confirmam que as mulheres são as que mais consomem medicamentos psicoativos, como os modeladores de apetite ${ }^{(6,7,11)}$. Dados coletados em São Paulo e Brasília revelaram prevalência significante do uso de moderadores de apetite entre as mulheres, principalmente de componentes como a anfetamina ${ }^{(6)}$. Uma pesquisa com estudantes da área da saúde no Nordeste do Brasil revelou que as anfetaminas estavam entre as substâncias ilícitas mais utilizadas, sendo seu uso mais comum entre os estudantes das classes sociais elevadas $(11,9 \%)^{(12)}$.

O uso de pílulas para emagrecimento é preocupante no Sudeste do Brasil. Um estudo transversal nessa região mostrou prevalência de 1,3\% do uso de moderadores de apetite na população adulta de ambos os sexos ${ }^{(7)}$. Além disso, observou-se, nos resultados desse estudo, que a maioria dos usuários $(81 \%)$ utilizava os moderadores de apetite de acordo com a prescrição do médico. Pesquisa conduzida em Porto Alegre, capital de Estado do sul do Brasil, também revelou o uso considerável de agentes farmacológicos com objetivo de controle do peso, incluindo laxantes $(8,5 \%)$ e diuréticos $(2,8 \%)^{(13)}$. Em um estudo conduzido em Florianópolis (SC), outro Estado do sul do país, as mulheres apresentaram consumo duas vezes maior de moderadores de apetite do que os homens ${ }^{(14)}$. Outras evidências obtidas no sul do Brasil confirmaram que as mulheres tendem a consumir mais medicamentos com anfetaminas do que os homens, principalmente com objetivo de moderar o apetite ${ }^{(15)}$.

Apesar dos medicamentos baseados em anfetamina serem os moderadores de apetite mais conhecidos, outras substâncias também são comumente utilizadas com o objetivo de reduzir peso. Todavia, existem poucos estudos sobre o uso adicional de moderadores de apetite. O fato é que a grande difusão do uso de anfetaminas no Brasil tem aumentado, afetando mulheres de todas as classes sociais. Tal questão sugere que a magnitude do abuso de moderadores de apetite é ainda mais significante.

\section{OBJETIVO}

Investigar o uso de diferentes tipos de moderadores de apetite em amostra de 300 estudantes matriculados nos cursos de ciências da saúde da Universidade de Caxias do Sul (UCS).

\section{METÓDOS \\ Participantes}

Investigou-se amostra de conveniência de 300 estudantes matriculados no primeiro semestre dos cursos de Biologia, Medicina, Enfermagem, Nutrição, Farmácia, Fisioterapia e Educação Física da UCS, localizada no sul do Brasil. Os participantes frequentaram as aulas de acordo com o calendário definido pela secretaria acadêmica da UCS. O principal critério de inclusão foi de o participante estar matriculado no primeiro semestre dos cursos de graduação em ciências da saúde da UCS. Todos os participantes eram falantes nativos da língua portuguesa.

\section{Coleta de dados}

Um membro do time de pesquisa frequentou todas as aulas do primeiro termo. Os estudantes eram informados sobre o objetivo do estudo durante um breve explicação estruturada no início da aula. Após apresentar o objetivo da pesquisa, os pesquisadores ofereciam um questionário para ser preenchido por todos os que desejavam participar. Os estudantes foram informados de que todos os dados fornecidos durante a pesquisa seriam tratados com confidencialidade e que, se desejado, poderiam se retirar da pesquisa a qualquer momento. Posteriormente, foi oferecido o Termo de Consentimento Livre Esclarecido para os participantes. Nenhuma assistência foi dada aos estudantes durante o preenchimento dos formulários. Após completarem as respostas, o que, em geral, levou de 10 a 15 minutos, todos os questionários e o respectivo Termo de Consentimento Livre Esclarecido foram coletados pelo pesquisador. Os estudantes que não participaram do estudo permaneceram na sala ou se retiraram. A coleta de dados foi concluída após 3 meses de investigação.

\section{Termo de Consentimento Livre e Esclarecido}

Este estudo foi aprovado pelo Comitê de Ética e Pesquisa da UCS (protocolo de pesquisa aprovado n. ${ }^{-}$41). Os procedimentos da pesquisa, incluindo coleta de dados, análise estatística e interpretação dos achados foram conduzidos entre 2004 e 2009. Todos os voluntários assinaram o Termo de Consentimento Livre e Esclarecido contendo a descrição dos procedimentos envolvidos no 
projeto. Todos os participantes completaram o questionário sob mínima orientação dos examinadores treinados, que seguiram procedimentos padrões.

\section{Instrumentos}

Os dados foram coletados por meio de questionário que incluía 24 itens para investigação de uma variedade de fatores relacionados ao uso de moderadores de apetite durante a vida. Uma lista de alopáticos e medicamentos alternativos incluiu estimulantes, inibidores seletivos da recaptação da serotonina (SSRIs), benzodiazepinas, laxativos, hipnóticos, hormônio lipotrófico, substâncias termogênicas (contendo L-carnitina, L-tirosina e L-fenilalanina), extratos herbais e "outros". Questões especificas exploraram o tipo de substância usada, enquanto outras questões abordaram a frequência do uso, a ocorrência de efeitos adversos e os fatores adicionais relacionadas ao uso da substância. O questionário utilizado, neste estudo, foi anteriormente validado em um estudo brasileiro comparativo( ${ }^{(7)}$. Coletou-se também a informação demográfica, incluindo gênero e idade.

\section{Registro dos dados e análise estatística}

Os questionários foram primeiramente verificados em relação ao seu total preenchimento e, subsequentemente, informatizados e armazenados em banco de dados organizados, utilizando planilhas Excel ${ }^{\circledR}$. Os dados foram convertidos ao formato Statistical Package for the Social Science $^{\circledR}$ (SPSS) e, então, analisados. Realizaram-se as análises de frequência do uso e a análise comparativa. Quando pertinente, duas tabelas de contingência com tabulação cruzada foram utilizadas para explorar estatisticamente os relacionamentos entre as duas variáveis. Esses procedimentos constituíram-se do teste do $\chi^{2}$ e do coeficiente de contingência $\mathrm{V}$ de Cramer.

\section{RESULTADOS}

\section{Informação demográfica}

Aproximadamente $75,3 \%(\mathrm{n}=226)$ da amostra foi constituída de mulheres. A média de idade foi de 26 anos (variação $=17-43$ ). As afiliações, de acordo com as áreas, foram Biologia $(8 \% ; n=24)$, Medicina $(8,33 \% ; n=25)$, Enfermagem $(16 \% ; n=48)$, Nutrição $(14 \% ; n=42)$, Farmácia $(14,66 \% ; n=44)$, Educação Física $(25 \%$; $=75)$ e Fisioterapia $(14 \%$; $=42)$.

\section{Uso de moderadores de apetite}

$\mathrm{Na}$ amostra, $15 \%(\mathrm{n}=45)$ dos participantes relataram usar moderadores de apetite pelo menos uma vez. O uso durante a vida de moderadores de apetite contendo tanto anfepramona quanto o femproporex foi de $4,66 \%(n=14)$, enquanto $10,66 \%(n=31)$ dos estudantes utilizou "outras" substâncias com objetivo de reduzir peso, incluindo SSRIs (3\%), extratos herbais $(1,66 \%)$, componentes classificados como termogêni$\cos (0,66 \%)$, benzodiazepinas $(0,33 \%)$, hormônio lipotrófico $(0,33 \%)$ e outros medicamentos não específicos $(4,66 \%)$. Dois participantes relataram o uso de mais de um tipo de substância.

A figura 1 mostra o uso de moderadores de apetite durante a vida por área: Enfermagem $(26,66 \%)$, Nutrição (24,44\%), Educação Física e Farmácia (17,77\%), Biologia e Fisioterapia (6,66\%), Medicina (não houve). A maioria dos estudantes que relatou o uso de pelo menos um medicamento moderador de apetite era mulher $(88,88 \%)$. A análise das duas tabelas de contingência demonstrou correlação significante entre a matrícula em cursos de graduação específicos e o uso de moderadores de apetite $\chi^{2}(6 ; n=300)=15,91, p=0,014$, $\mathrm{V}$ de Cramer $=0,23$.

Em termos de fonte de aconselhamento para uso de moderadores de apetite, $28,88 \%(n=13)$ dos estudantes se automedicavam, 13,32\% $(n=6)$ mencionaram aconselhamento por um membro familiar, farmacêutico, amigo ou outro. Por outro lado, $57,7 \%(n=26)$ da amostra recebeu aconselhamento e prescrição de um médico. Além disso, quase um quarto dos participantes $(24,4 \%)$ que fizeram uso de moderadores consultou um médico nos 6 meses precedentes.

A prevalência de reações adversas aos moderadores de apetite foi significante. Cerca de 10 a $20 \%$ dos voluntários suspenderam o uso dos agentes devido aos efeitos adversos, como irritabilidade, nervosismo, insônia, tristeza, sinais e sintomas físicos emergenciais.

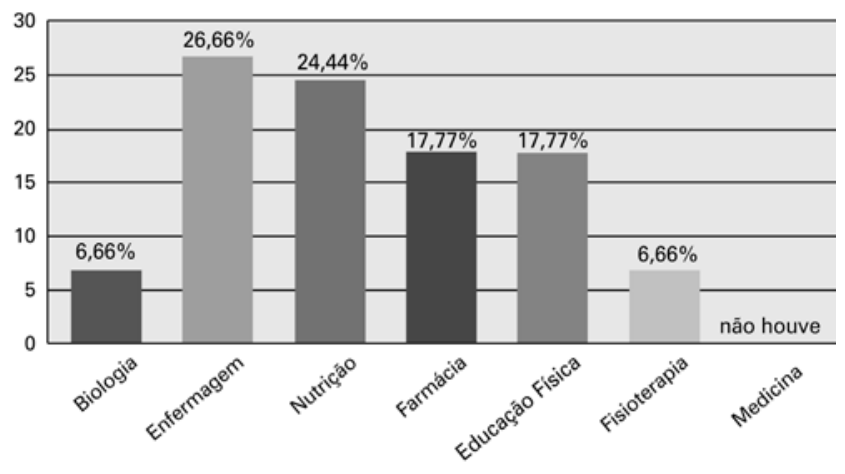

Figura 1. Porcentagem do uso de moderadores de apetite entre estudantes de graduação em diferentes cursos em ciências da saúde 
Apenas 11,11\% $(\mathrm{n}=5)$ daqueles que relataram o uso de moderadores de apetite continuaram a consultar o médico, enquanto 46,67\% $(\mathrm{n}=21)$ relataram descontinuar o acompanhamento. O restante dos participantes não especificou essa informação. Dois usuários de moderadores de apetite $(4,44 \%)$ relataram aumento da dose sem supervisão médica, enquanto 19 estudantes $(42,22 \%)$ não indicaram nenhuma resposta relacionada ao aumento da dose. A média de duração do uso foi de 99,48 dias por usuário. Quatro participantes relataram utilizar moderadores de apetite com frequência.

Além disso, 48,88\% $(n=22)$ daqueles que relataram o uso de moderadores de apetite o realizaram por meio de prescrição médica, enquanto $17,77 \%(\mathrm{n}=8)$ compraram a medicação em farmácia sem prescrição. Onze estudantes relataram "outros" como meio de obter a medicação.

\section{DISCUSSÃO}

Neste estudo, o uso de moderadores de apetite foi relatado entre os estudantes matriculados nos cursos de graduação em ciências da saúde do primeiro semestre da UCS. Os resultados relatados demonstraram que o uso de moderadores de apetite foi consideravelmente prevalente na amostra investigada e variou de acordo com a área acadêmica do estudante. $\mathrm{O}$ fato de que, no futuro, alguns estudantes analisados se tornarão profissionais de saúde com acesso fácil a essas substâncias é preocupante.

Porém, deve-se considerar que os estudantes estavam na fase inicial de suas carreiras, o que pode sugerir que a alta aceitação, de uma fração deles, dos moderadores de apetite está relacionada a visões pré-acadêmicas. $\mathrm{O}$ modelo transversal deste estudo não permitiu interferências em relação aos possíveis efeitos atenuantes da educação acadêmica no uso de moderadores de apetite. Pode-se supor que os estudantes em ciências da saúde podem se expor aos moderadores de apetite devido ao interesse às questões relacionadas à saúde.

Neste estudo, os estudantes de medicina foram uma exceção à tendência da prevalência do uso dessas substâncias entre os futuros médicos. Esses resultados contrastam com evidências anteriores, que revelaram nível significante do uso de medicamentos psicoativos entre os estudantes de medicina na UCS ${ }^{(16)}$. Em contraste com esta pesquisa, um estudo anterior, com estudantes de todos os anos do curso de medicina, não apresentou desproporção significativa entres os gêneros ${ }^{(16)}$. É possível que deficiências na metodologia impediram a detecção do uso dessas substâncias entre os estudantes de medicina. Por outro lado, o não relato do uso de mode- radores de apetite entre estudantes de medicina pode sugerir que os futuros médicos serão menos inclinados a prescrever moderadores de apetite a seus pacientes, a não ser que a tendência de prescrever tais substâncias se desenvolva durante os anos de formação. No estudo mencionado anteriormente, as anfetaminas foram o único tipo de substância que os participantes relataram uso anterior antes da residência ${ }^{(16)}$, um achado que concorda com as evidências adicionais demonstrando uso mais frequente de medicamentos psicotrópicos durante os últimos anos do curso de medicina ${ }^{(17,18)}$.

A maioria dos usuários de moderadores de apetite foi do gênero feminino, o que confirma achados anteriores no Brasil da prevalência do uso dessas substâncias entre mulheres ${ }^{(11,19)}$. As jovens brasileiras são muito preocupadas com seu corpo $^{(20)}$. A cultura da magreza tem sido considerada a principal causa para o uso de moderadores de apetite de modo "epidêmico" ou desnecessário ${ }^{(11,19)}$. No Brasil, uma análise com mais de 40 mil prescrições médicas na cidade de São Paulo revelou que tais medicamentos foram prescritos 10 vezes mais para mulheres do que para homens ${ }^{(21)}$. Estudo anterior, conduzido no Brasil ${ }^{(11)}$, revelou que os moderadores de apetite foram prescritos mesmo sem qualquer indicação de obesidade. Resultados similares mostraram que $25,1 \%$ das pessoas utilizando pílulas para perda de peso estavam abaixo do índice de massa corporal mínimo recomendado, indicando, portanto, uso inapropriado dessas prescrições ${ }^{(22)}$.

A prescrição do uso de moderadores de apetite permanece uma prática comum no Brasil ${ }^{(10,11)}$ principalmente no Rio Grande do Sul, estado da região sul do Brasil $^{(23)}$. A maioria dos usuários de moderadores de apetite seguiu o aconselhamento médico quando administraram esses medicamentos por contra própria. Ainda, quase um terço de todos os usuários de moderadores de apetite tomou essas medicações sem avaliação ou prescrição médica. Mais de um quarto dos usuários relatou comprar moderadores de apetite diretamente nas farmácias e sem guia médica. A prescrição inapropriada de moderadores de apetite tem sido relatada em outros países ${ }^{(8,24)}$. O risco associado ao uso de moderadores de apetites tem levado alguns países a adotar medidas restritivas para prescrição dessas substâncias ${ }^{(25)}$.

A duração máxima recomendada para terapia com moderadores de apetite é de 3 meses ${ }^{(26,27)}$ Neste estudo, a média de uso dos moderadores de apetite foi de quase 100 dias, o que excede a recomendação da Organização Mundial da Saúde. Em estudo recente, muitas substâncias utilizadas por estudantes apresentaram efeito estimulante no Sistema Nervoso Central, sendo que as reações adversas mais comuns relatadas foram: agita- 
ção, nervosismo, irritabilidade e insônia. Essas reações podem ser tão intolerantes, que a maioria dos pacientes interrompeu o uso da medicação. $\mathrm{Na}$ amostra deste estudo, grande proporção dos usuários interrompeu o uso de moderadores de apetite devido aos efeitos adversos. Tais efeitos podem também incluir dependência do medicamento, aumento da pressão sanguínea, hipertensão pulmonar, valvulopatia cardíaca e, eventualmente, ilusões paranoides ${ }^{(28-31)}$.

No Brasil, muitos indivíduos tentam perder peso tomando medicamentos, como as anfetaminas, por meio de fórmulas contendo diversas substâncias ${ }^{(11,19)}$. Essas combinações, chamadas de "manipulação" de moderadores de apetite, são feitas por farmácias autorizadas e contêm anfetaminas e benzodiazepinas além de diuréticos, agentes tirodianos, laxantes e plantas medicinais $^{(7,11,19)}$. Neste estudo, não se investigaram as fórmulas manipuladas. O fato de que $17,77 \%$ dos usuários obtiveram os moderadores de apetite diretamente na farmácia sugere que o uso dessas fórmulas também pode ter ocorrido nesta amostra.

A prática de distribuição de medicamentos anfetamínicos combinados com outras substâncias foi difundida no Brasil ${ }^{(32)}$. Na verdade, o uso de pílulas para emagrecimento cruzou os limites geográficos do país, tornando-se também comum entre os imigrantes nos Estados Unidos. Os resultados de uma pesquisa cega $(n=307)$ conduzida em uma clínica e em duas igrejas com mulheres imigrantes, com idade entre 18 e 50 anos, morando nos Estados Unidos, revelaram que $18 \%$ das clientes da clínica e 9\% das frequentadoras da igreja utilizavam pílulas para emagrecimento ${ }^{(33)}$. Quase dois terços dos usuários dessas pílulas relataram efeitos adversos. Mulheres solteiras, com nível superior e aconselhadas por médico americano a perder peso estavam altamente associadas ao uso de pílulas para emagrecimento ${ }^{(33)}$.

Apesar da resolução do Conselho Federal de Medicina do Brasil ${ }^{(34)}$, que mais tarde foi ratificada pelo Ministério da Saúde ${ }^{(35)}$, proibindo a prescrição de substâncias combinadas contendo anfetaminas, benzodiazepinas, diuréticos, derivados hormonais e laxantes, ainda continua a distribuição dessas substâncias. Em outubro de 2011, a Agência Nacional de Vigilância Sanitária (ANVISA) publicou resolução proibindo a prescrição, o fornecimento e a distribuição de anfepramona, mazindol e fenproporex ${ }^{(36)}$. Evidências recentes sugerem que, após o endurecimento dessa legislação, houve redução progressiva na distribuição de moderadores de apetite banidos no Brasil ${ }^{(37)}$.

Este estudo apresentou algumas possíveis limitações. Primeiro, a pesquisa foi desenvolvida em uma única universidade, o que limita a possibilidade dos resultados serem mais gerais. Segundo, é possível que muitos estudantes que consumiram os moderadores de apetite não preencheram a pesquisa honestamente, fato que pode ter comprometido os resultados. Terceiro, foi utilizada, no estudo, amostra de conveniência ${ }^{(38)}$. Amostras de conveniência podem sofrer diversos vieses, incluindo baixa ou alta representatividade de grupos específicos. Tais questões podem limitar a generalização e interferir na população total, comprometendo a validade externa dos achados do estudo. Por outro lado, a escolha de amostra de conveniência foi baseada em diversas vantagens, incluindo a natureza direta do procedimento de amostragem, o baixo custo e o tempo reduzido, também como a oportunidade de reunir dados e informações que não são possíveis de coletar por meio de técnicas de amostragem probabilística e mais sistematizadas. Por fim, considerando que este estudo foi conduzido antes da publicação da resolução da ANVISA em 2011, os resultados aqui apresentados podem não representar rigorosamente os padrões atuais quanto ao uso de moderadores de apetite no Brasil.

\section{CONCLUSÃO}

Neste estudo, houve preocupação quanto ao nível do uso de moderadores de apetite entre os estudantes de graduação matriculados no primeiro semestre dos cursos de ciências da saúde da UCS. Os resultados obtidos confirmaram que o uso dessa substância foi mais significativo entre as mulheres. A maioria relatou utilizar moderadores de apetite após aconselhamento médico e muitos interromperam a medicação devido aos efeitos adversos. A média de duração do tratamento com moderadores de apetite foi superior a 3 meses. Outros estudos comparativos são necessários para elucidar se estudantes matriculados nas áreas de ciências da saúde apresentam níveis mais elevados do uso de moderadores de apetite do que estudantes de outras áreas. Estudos prospectivos também ajudariam a estabelecer se o acesso a informação relacionada à saúde, ao contrário do que é esperado, facilita ou não o uso de moderadores de apetite. Os achados apresentados neste estudo justificam a necessidade de outras pesquisas no assunto.

\section{REFERÊNCIAS}

1. James WP. WHO recognition of the global obesity epidemic. Int J Obes (Lond) 2008; 32 Suppl 7:S120-6.

2. Hebebrand J, Hinney A. Environmental and genetic risk factors in obesity Child Adolesc Psychiatr Clin N Am. 2009;18(1):83-94.

3. Alemany $M$, Remesar $X$, Fernández-López JA. Drug strategies for the treatment of obesity. IDrugs. 2003;6(6):566-72. 
4. Riggs P. Non-medical use and abuse of commonly prescribed medications. Curr Med Res Opin. 2008;24(3):869-77.

5. Vanbuskirk KA, Potenza MN. The Treatment of Obesity and Its Co-occurrence with Substance Use Disorders. J Addict Med. 2010;4(1):1-10.

6. Nappo SA, Tabach R, Noto AR, Galduróz JC, Carlini EA. Use of anorectic amphetamine-like drugs by Brazilian women. Eat Behav. 2002;3(2):153-65.

7. de Lima MS, Béria JU, Tomasi E, Mari JJ. Use of amphetamine-like appetite suppressants: a cross-sectional survey in Southern Brazil. Subst Use Misuse. 1998;33(8):1711-9

8. Sritipsukho P, Pacharapaisan N. Inappropriate weight management among Thai women consuming anorectics prescribed by private clinics in Bangkok. J Med Assoc Thai. 2004;87(12):1477-81.

9. Galduróz JC, Noto AR, Nappo SA, Carlini EL. First household survey on drug abuse in Sao Paulo, Brazil, 1999: principal findings. São Paulo Med J. 2003; 121(6):231-7.

10. Stempliuk Vde A, Barroso LP, Andrade AG, Nicastri $S$, Malbergier A. Comparative study of drug use among undergraduate students at the University of Sao Paulo--Sao Paulo campus in 1996 and 2001. Rev Bras Psiquiatr. 2005;27(3):185-93.

11. Nappo SA, de Oliveira EM, Morosini S. Inappropriate prescribing of compounded antiobesity formulas in Brazil. Pharmacoepidemiol Drug Saf. 1998;7(3):207-12.

12. Lucas AC, Parente RC, Picanço NS, Conceição DA, Costa KR, Magalhaes $I R$, et al. [Use of psychoactive drugs by health sciences undergraduate students at the Federal University in Amazonas, Brazil]. Cad Saude Publica. 2006;22(3):663-71. Portuguese.

13. Nunes MA, Barros FC, Anselmo Olinto MT, Camey S, Mari JD. Prevalence of abnormal eating behaviours and inappropriate methods of weight control in young women from Brazil: a population-based study. Eat Weight Disord. 2003;8(2):100-6.

14. Baus J, Kupek E, Pires M. [Prevalence and risk factors associated with drug use among school students, Brazil]. Rev Saude Publica. 2002;36(1):40-6. Portuguese.

15. Barcellos AP, Paggi AP, Silva DB, Campagnolo Ml, Dieterich MD, Santos RLR, et al. Padrão de consumo de anfetaminas entre universitários de Porto Alegre. Rev Psiquiatr Rio Gd Sul. 1997;19(3):161-9.

16. Boniatti MM, Zubaran C, Panarotto D, Delazeri GJ, Tirello JL, Feldens Mde 0 , et al. The use of psychoactive substances among medical students in southern Brazil. Drug Alcohol Rev. 2007;26(3):279-85.

17. Andrade AG, Bassit AZ, Mesquita AM, Fukushima JT, Gonçalves EL. Prevalência de uso de drogas entre alunos da Faculdade de Medicina da Universidade de São Paulo (1991-1993). Rev ABP-APAL. 1995;17(2):41-6.

18. Kerr-Corrêa F, Andrade AG, Bassit AZ, Boccuro NM. Uso de álcool e drogas por estudantes de medicina da UNESP. Rev Bras Psiquiatr. 1999;21:95-100.

19. Nappo SA. Consumption of anorexigenic amphetamine-like drugs (diethylpropion, fenproporex and mazindol) and of $d, 1$-fenfluramine in Brazil during the years of 1988 and 1989. Pharmacoepidemiol Drug Saf. 1996:5(1):19-25.
20. Fonseca Vde M, Sichieri R, da Veiga GV. [Factors associated with obesity among adolescents]. Rev Saude Publica. 1998;32(6):541-9. Portuguese.

21. Noto AR, Carlini EA, Mastroianni PC, Alves VC, Galduroz JC, Kuroiwa W, et al Analysis of prescription and dispensation of psychotropic medications in two cities in the State of São Paulo, Brazil. Rev Bras Psiquiatr. 2002;24(2):68-73.

22. Khan LK, Serdula MK, Bowman BA, Williamson DF. Use of prescription weight loss pills among U.S. adults in 1996-1998. Ann Intern Med. 2001;134(4):282-6.

23. Mota DM, Silva-Jr GG. [Evidence for the use of appetite suppressant drugs in Brazil: a pharmacoeconometric study]. Rev Assoc Med Bras. 2012;58(1):88-94.

24. Stafford RS, Radley DC. National trends in antiobesity medication use. Arch Intern Med. 2003;163(9):1046-50.

25. Colman E. Anorectics on trial: a half century of federal regulation of prescription appetite suppressants. Ann Intern Med. 2005;143(5):380-5

26. WHO. Anorectic agents containing amphetamines - restricted indications WHO Pharmaceuticals Newsletter. 1995;8.

27. WHO. Anorectic agents - restricted use. WHO Pharmaceuticals Newsletter 1997;5-6.

28. Carney MW. Diethylpropion and psychosis. Clin Neuropharmacol. 1988;11(2): 183-8.

29. Silverstone T, Goodall E. Centrally acting anorectic drugs: a clinical perspective. Am J Clin Nutr. 1992;55(1 Suppl):211S-4S

30. Abenhaim L, Moride Y, Brenot F, Rich S, Benichou J, Kurz X, et al. Appetitesuppressant drugs and the risk of primary pulmonary hypertension. International Primary Pulmonary Hypertension Study Group. N Engl J Med. 1996;335(9):609-16.

31. Jick H. Heart valve disorders and appetite-suppressant drugs. JAMA. 2000; 283(13):1738-40

32. Nappo S, Carlini E, Araújo M, Moreira L. Prescription of anorectic and benzodiazepine drugs through notification B prescriptions in Natal, Rio Grande do Norte, Brazil. Braz J Pharm Sci. 2010;46(2):297-303.

33. Cohen PA, McCormick D, Casey C, Dawson GF, Hacker KA. Imported compounded diet pill use among Brazilian women immigrants in the United States. J Immigr Minor Health. 2009;11(3):229-36.

34. Conselho Federal de Medicina. Resolução CFM no 1.477/97 [Internet]. Available from: http://www.portalmedico.org.br/resolucoes/cfm/1997/1477_1997.htm

35. Agência Nacional de Vigilância Sanitária (ANVISA). Portaria N. ${ }^{0} 344$, de 12 de Maio de 1998 [Internet]. Disponível em: http://www.anvisa.gov.br/ scriptsweb/anvisalegis/VisualizaDocumento.asp?|D $=939 \& V e r s a 0=2$

36. Agência Nacional de Vigilância Sanitária (ANVISA). Resolução- RDC No 52, de 6 de Outubro de 2011 [Internet]. Disponível em: http://www.in.gov.br/visualiza/ index.jsp?data $=10 / 10 / 2011$ \&jornal $=1$ \&pagina $=55$ \&totalArquivos $=128$

37. Castro L, Farias Junior G, Teixeira F, Vieira J, Maia C. Impact of health legislation on the sale of anorectics in a city in the Amazon region. Acta Amazon. 2012;42:215-20.

38. Kalsbeek W, Heiss G. Building bridges between populations and samples in epidemiological studies. Annu Rev Public Health. 2000;21:147-69. 